\title{
Anticorrelation of photoluminescence from gold nanoparticle dimers with hot-spot intensity
}

Dmitry Sivun, Cynthia Vidal, Battulga Munkhbat, Nikita Arnold, Thomas A. Klar, and Calin Hrelescu*

Institute of Applied Physics, Johannes Kepler University Linz, 4040 Linz, Austria

*calin.hrelescu@jku.at

\section{Supporting Information}

\section{A) Experimental setup}

The experimental setup is shown in Figure S1. All optical measurements are carried out on an inverted optical microscope Olympus IX-71. The microscope was modified in order to have a simultaneous access to darkfield scattering measurements (Schott KL1500 lamps were used as a source), and photoluminescence measurements. The excitation source for photoluminescence measurements was a $405 \mathrm{~nm}$ linearly polarized continuous wave laser (LDH-D-C-405) from PicoQuant providing an intensity of $\mathrm{I} \approx 6 \mathrm{~kW} / \mathrm{cm}^{2}$ in the focal area. The linear polarization was along the long axes (and the connecting axis) of the BP dimers. Excitation and detection were performed by the same UPLSAPO 40x (NA = 0.95) Olympus objective lens. The scattered or PL light was coupled to a $105 \mu \mathrm{m}$ core fiber, spectrally dispersed by a Zolix Omni- $\lambda 3006$ spectrometer and detected by a CCD (Andor iDUS 420). The manipulation steps and topography measurements were carried out by an atomic force microscope (JPK Instruments, NanoWizard 3) 
placed on top of the inverted microscope. For both, shifting and seesawing the BPs, AFM probes from Nanosensors (model: PPP-FMR) were used with a force constant around C $=3 \mathrm{~N} / \mathrm{m}$. The sample stage and the AFM head each have their separate, three axis piezo control with capacitive feedback.

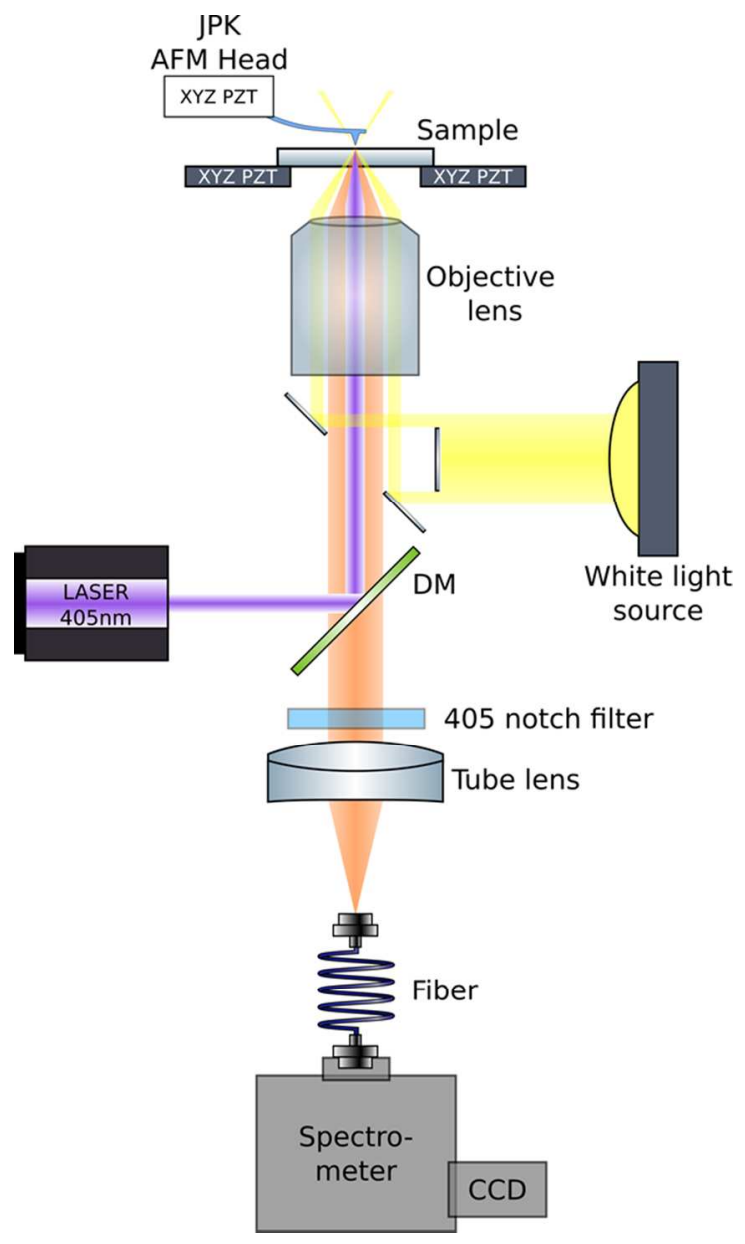

Figure S1. Scheme of the experimental setup.

\section{B) Characterization of single bipyramids}

Before forming a dimer, two bipyramids with similar scattering signals where identified using dark field scattering spectroscopy. At that time, the individual BPs where well separated, so that the single particle spectra where not compromised by electromagnetic coupling to other particles. 
First, the topography was imaged using tapping mode AFM, which proved that the NPs are indeed bipyramids, as well as that the surrounding of the BPs is clear of any other particles (Inset of Figures S2 a,b). In fact, Figures S2 a and b show the scattering and PL spectra of exactly those two NPs that where later nanomanipulated to form the dimer used in the experiments described in the main text (Figures 3,4).

The single BP photoluminescence is always blueshifted compared to the scattering spectra, with a blueshift ranging from 8 to $10 \mathrm{~nm}$. Additionally, there is a clear asymmetry in the photoluminescence spectra, where the signal at short wavelengths is more pronounced than the signal at long wavelengths, similar to what is observed for dimers, see main text.
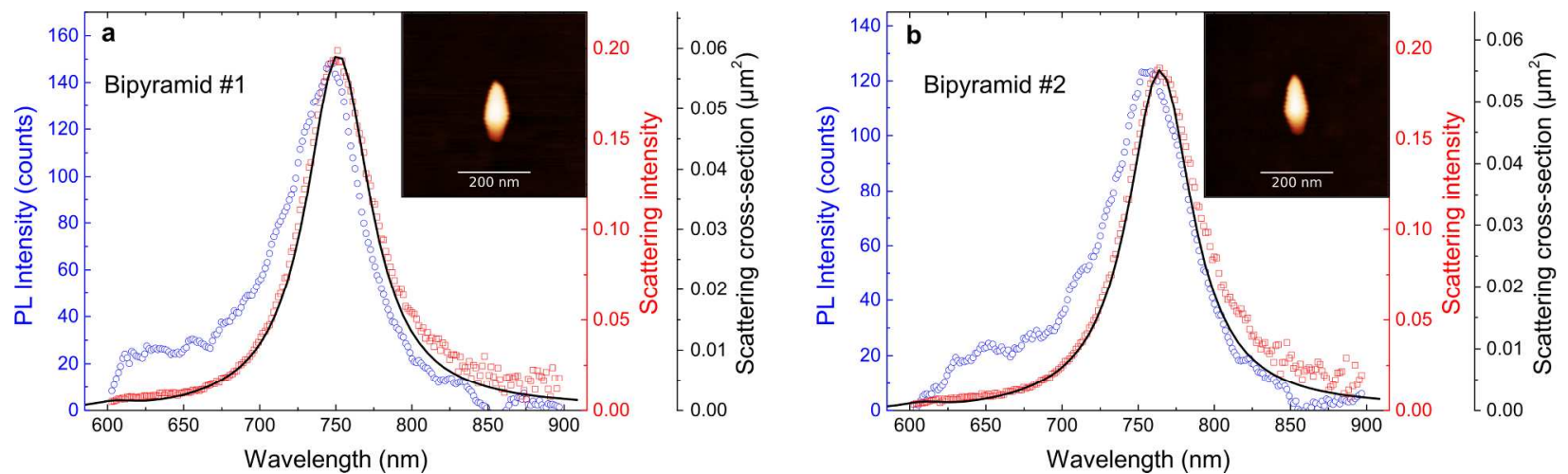

Figure S2. Optical characteristics of the two BPs before forming the dimer used in experiments described in the main text. Open red squares and blue circles correspond to experimentally measured scattering and photoluminescence (PL) spectra, respectively. Solid black line: numerically calculated scattering cross sections. Insets are AFM images of the two BPs, taken with an AFM in tapping mode. 


\section{C) Formation of dimers of bipyramids}

The two BPs characterized in section B were mechanically positioned next to each other using AFM nano-manipulation ${ }^{1-4}$ and subsequently aligned so that their individual long axes are collinear to form dimers with controlled center-to-center distances. Due to their morphology, especially due to the pentagonal cross-section, individual BPs deposited on a substrate are lying on one of the ten facets so that one BP apex is located on the substrate, while the other BP's tip is pointing upwards. This peculiar property allows us to arrange the two BPs in the dimer in three different configurations while keeping the center-to-center distance between the two BPs constant. In contrast to other reports on the scattering of dimers consisting of BPs, where several dimers were formed in solution with fixed apex-to-apex distances and subsequently spin cast on a substrate, ${ }^{5}$ our approach allows to investigate the same pair of BPs at different distances from each other and in the three different tip-configurations. We denote the configurations corresponding to the vertical positions of the two BPs' tips pointing towards each other in the dimer as Up-Up, Up-Down and Down-Down, respectively. 


\section{D) Retrieval of geometric parameters for simulations}
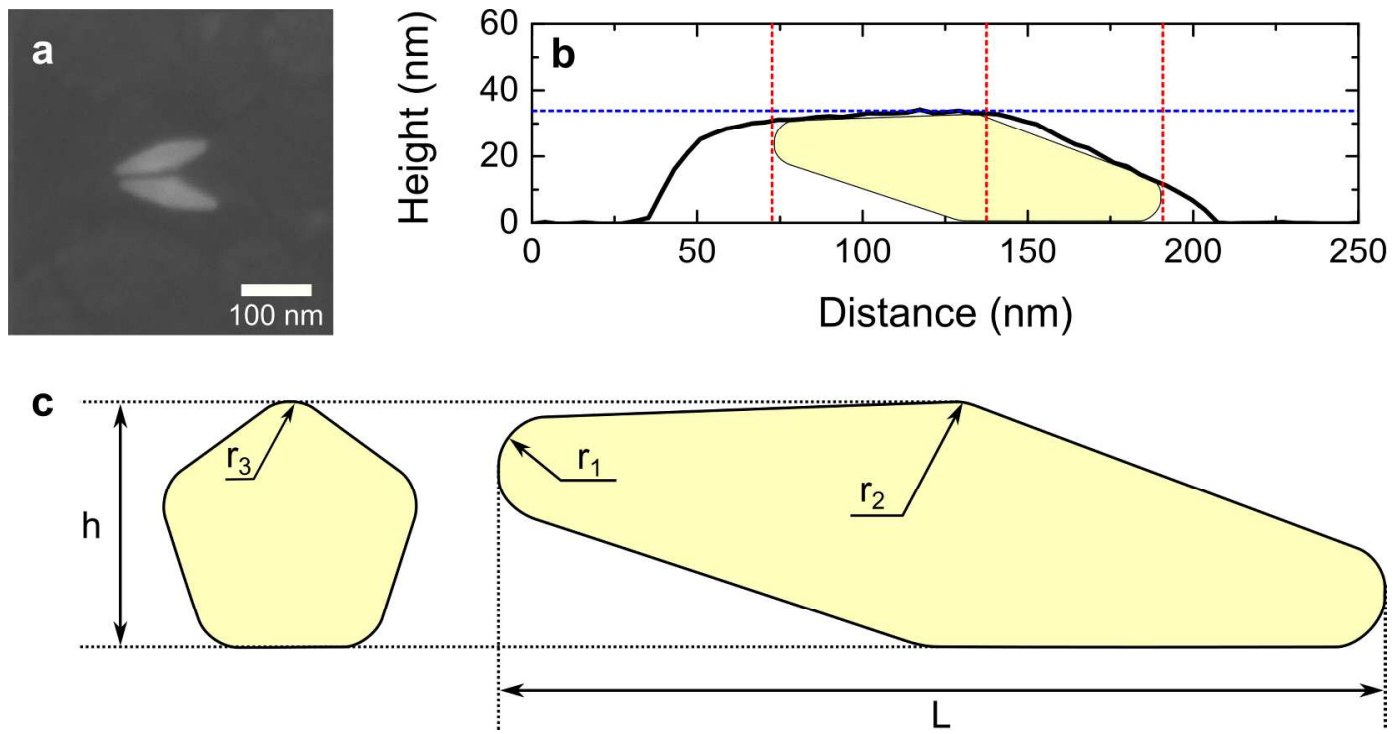

Figure S3. a) SEM image of two BPs from the same batch of BPs as used in the experiment. b) Cross section from an AFM image (Inset in Figure S2a), from which the height of the BP was retrieved as well as other geometrical parameters needed for calculating the geometry and interparticle distance. c) Geometrical parameters of a BP used in numerical simulation. $L$ is the length of a BP, $h$ is the height and $r_{1}, r_{2}$, and $r_{3}$ are radii of curvature introduced in order to smooth edges.

All simulations were performed in frequency domain with the commercial finite element solver COMSOL ${ }^{\mathrm{TM}}$, version 4.4 , using the Wave Optics package. The polarization of the incident electric field was always parallel to the long axis of a bipyramid dimer axis, and normal incidence was assumed. The dielectric function of gold was taken from Johnson and Christy ${ }^{6}$. The simulation model consisted of a glass substrate with refractive index $n=1.523$, topped with bipyramidal NPs, and air with $n=1$. First of all a single bipyramid was simulated with geometrical parameters taken from SEM and AFM measurements (Fig. S3 a,b and inset of Fig. $\mathrm{S} 2$ ). The SEM image gives an approximate number for the length $L$ and the angle of inclination 
of the BPs, while the AFM measurements revealed the absolute height $h$. In order to fit the simulated scattering spectra to the experimental ones, the length of the BP model was fine-tuned. The final geometrical parameters retrieved for the two BPs used to form the dimer in the main text are: $L=122.6 \mathrm{~nm}$ and $h=35.3 \mathrm{~nm}$ for the first BP and $L=121.8 \mathrm{~nm}$ and $h=33.5 \mathrm{~nm}$ for the second BP. Note that the calculations were carried out respecting this tiny difference in the size of the two BPs. For both BPs, the radii of curvature of the tips, as well as radii of edge smoothing (Fig. S3c) were $r_{1}=r_{3}=6 \mathrm{~nm}$, and $r_{2}=12 \mathrm{~nm}$, respectively (Fig. S3 c).

\section{E) Tip Deconvolution}

In order to be able to retrieve the interparticle distance from AFM topography measurements, the profile of the AFM tip and the profile of BPs need to be deconvolved. This way, it is possible to retrieve the real geometrical parameters of a dimer of BPs from the measured AFM topography. There are several reports dedicated to this problem, ${ }^{2,7}$ but these describe only the case of symmetric particles like spheres. In case of BPs lying on one of the facets, the structure is asymmetric, and the convolution near the upper tip of a BP is different from the convolution near the lower tip of a BP.

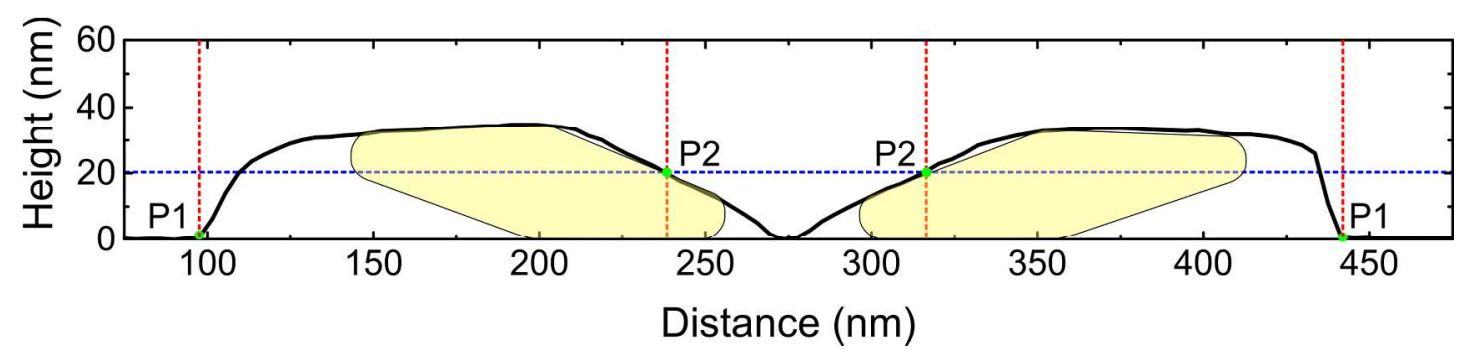

Figure S4. Topographical height profile of the BPs dimer. The black line is the measured height profile. The yellow bipyramids show the assumed positions of the BPs. 
Figure S4 shows a height profile obtained from an AFM topography image of a BP dimer. The solid black line is a measured height profile, the yellow BPs fitted inside of it is the profile of the BP used for simulation. As we can see from figure S4, the tip-convoluted lengths of the BPs appear much longer than the real length $L$, and convolution of the AFM tip is much more pronounced on the side of the upper tip. Even worse, the convolution with the AFM tip could change during experiments (i.e. after some manipulation steps due to slight changes of the tip profile). Therefore, the determination of the distance between BPs is not possible using points P1 in Fig. S4. Generally, one can expect an increase of the convolution with the AFM tip with an increase of the number of manipulation steps, which will lead to a growing error in the interparticle distance in such case. In order to avoid this error, we measure the interparticle distance at convolution-free points of the height profile. Such points are for example the points

P2 where the inclined slope of the tips reaches a certain height (e.g. $20 \mathrm{~nm}$, dashed blue horizontal line), as height measurements are by far more accurate in AFM than lateral measurements. The closest position of such selected points, which could be achieved during nanomanipulation, determines the zero distance $(d=0)$.

\section{F) Retrieval of positions of PL side peaks, supported by higher order plasmons:}

Three Lorentzians were fit in order to get the positions of the main peak and two weaker signatures in the photoluminescence spectra. As a Lorentzian is well defined in energy rather than wavelength space, the abscissas were first converted to energy scale. Then three Lorentzian curves were fit. One example of fitting is illustrated in figure S5. The obtained peak positions where then calculated back to wavelengths in order to obtain the data shown in figure 5 of the main text. 


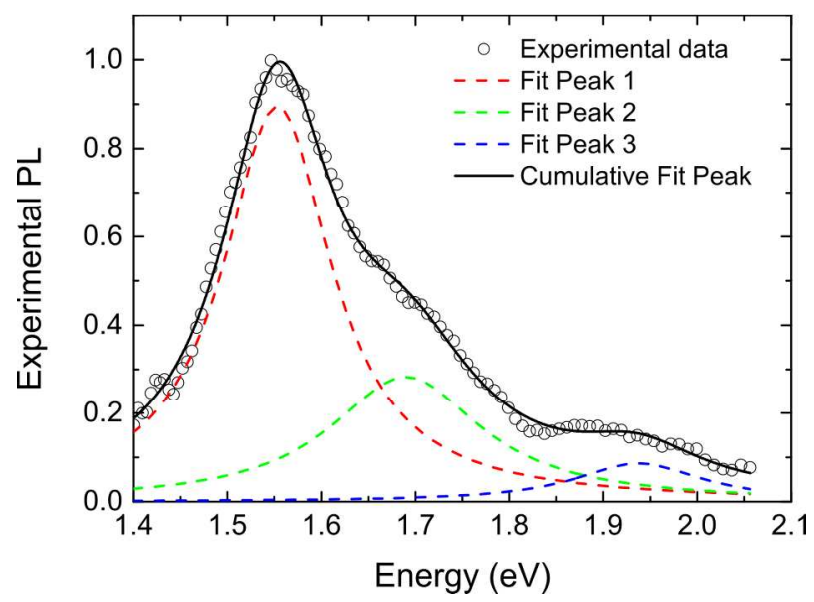

Figure S5: Black open circles show experimentally measured PL- spectra for the UpDown configuration and an interparticle distance $d=3 \mathrm{~nm}$. Red, green, and blue dashed lines indicate the Lorentzian fits for dipolar, quadrupolar, and higher order plasmonic modes respectively. The black solid line shows the cumulative fit.

\section{References}

(1) Schaefer, D. M.; Reifenberger, R.; Patil, A.; Andres, R. P. Appl. Phys. Lett. 1995, 66, 1012.

(2) Junno, T.; Deppert, K.; Montelius, L.; Samuelson, L. Appl. Phys. Lett. 1995, 66, $3627-$ 3629.

(3) Hansen, L. T.; Kühle, A.; Sørensen, A. H.; Bohr, J.; Lindelof, P. E. Nanotechnology 1998, $9(4), 337$.

(4) Bek, A.; Jansen, R.; Ringler, M.; Mayilo, S.; Klar, T. A.; Feldmann, J. Nano Lett. 2008, 8, 485-490.

(5) Malachosky, E. W.; Guyot-Sionnest, P. J. Phys. Chem. C 2014, 118 (12), 6405-6412.

(6) Johnson, P. B.; Christy, R. W. Phys. Rev. B 1972, 6 (12), 4370-4379.

(7) Kim, S.; Shafiei, F.; Ratchford, D.; Li, X. Nanotechnology 2011, 22 (11), 115301. 